\title{
Multichannel quantum defect theory of photodissociation in $\mathrm{H}_{2}$
}

\author{
J.Zs. Mezei ${ }^{1,2, a, *}$, I.F. Schneider ${ }^{2}$ and Ch. Jungen ${ }^{1,3}$ \\ ${ }^{1}$ Laboratoire Aimé-Cotton, CNRS/Université Paris Sud, Bât. 505, 91405 Orsay, France \\ ${ }^{2}$ Laboratoire Ondes et Milieux Complexes UMR-6294 CNRS and Université du Havre, \\ 25, rue Philippe Lebon, BP. 540, 76058 Le Havre, France \\ ${ }^{3}$ Department of Physics and Astronomy, University College London, London WC1E 6BT, UK \\ * Also at: Institute for Nuclear Research, Hungarian Academy of Sciences, Debrecen, Hungary
}

\begin{abstract}
The predissociation profiles of the $R(0), R(1)$ and $R(2)$ lines of the $3 p \pi^{1} \Pi_{u}^{+}, v=$ $3 \leftarrow X^{1} \Sigma_{g}^{+}, v^{\prime \prime}=0$ absorption transition in $\mathrm{H}_{2}$ are calculated in a multichannel quantum defect theory approach, implemented on the basis of state-of-the-art potential energy curves (PEC) from the Wolniewicz group. It is shown that the profiles may be very accurately represented by the Fano profile formula although the resonance parameters thus determined do not exactly coincide with the meaning given to them in Fano's original derivation. The recent high-resolution spectrum of the same transitions taken with the SOLEIL synchrotron is well reproduced by the calculations.
\end{abstract}

\section{Introduction}

It has been known for a long time that the levels $v \geq 3$ of the $3 p \pi D^{1} \Pi_{u}^{+}$state of diatomic hydrogen $\mathrm{H}_{2}$ are strongly predissociated by the vibrational continuum of the $3 p \sigma B^{\prime}{ }^{1} \Sigma_{u}^{+}$state which correlates with the $\mathrm{H}(1 s)+\mathrm{H}(n=2)$ atomic states [1]. The interaction between the bound and the continuum states leads to broadening of the bound states which therefore have the character of resonances and appear with characteristic line profiles in the photoabsorption spectrum of ground state $\mathrm{H}_{2}$. This predissociation has been the first case in a molecule where asymmetric line profiles were observed of a type named after Beutler and Fano, who studied them in atoms experimentally and theoretically in the 1930's $[2,3]$. Numerous experimental and theoretical papers have been devoted to the study of the $D$ state predissociation resonances. Over the years, the spectral resolution has been improved and the theoretical methods used have been refined. However, practically all studies, whether experimental or theoretical, made use of Fano's well-known profile formula [4], by determining or predicting the width and shape parameters in terms of which the formula is expressed. We mention here, in order to place our subject into a broader context, that the Fano profile formula has received some criticism in its time [5] because it does not leave the total transition probability invariant (as one should expect on general grounds), and

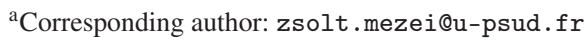

This is an Open Access article distributed under the terms of the Creative Commons Attribution License 4.0, which permits unrestricted use, distribution, and reproduction in any medium, provided the original work is properly cited. 
that the subject of time resolved Fano resonances associated with impulsive ionization by optical pulses has recently aroused interest $[6,7]$.

The Fano formula [4] is a generalization of the familiar Breit-Wigner expression [8] for resonant scattering. It expresses the cross section as

$$
\sigma(\epsilon)=\sigma_{b}+\sigma_{c} \frac{(q+\epsilon)^{2}}{1+\epsilon^{2}} .
$$

Here, $\sigma(\epsilon)$ is the resonant (perturbed) cross section while $\sigma_{c}$ is the unperturbed "flat" continuum cross section, taken here as constant, and $\sigma_{b}$ is a constant background contribution. $\epsilon$ and $q$ are dimensionless parameters defined, respectively, as

$$
\begin{aligned}
\epsilon & =\frac{E-E_{r}}{\frac{1}{2} \Gamma} \\
q & =\frac{\left\langle\psi_{d}|D| \psi_{0}\right\rangle\left(\frac{1}{2} \pi \Gamma\right)^{-1 / 2}}{\left\langle\psi_{c}|D| \psi_{0}\right\rangle},
\end{aligned}
$$

where $\epsilon$ is the reduced energy, $E$ is the energy, $E_{r}$ is the resonance energy, and $\Gamma$ is the resonance width. The shape parameter $q$ is defined as the ratio of the unperturbed transition moments from the ground state $\psi_{0}$ to the discrete level and to the continuum, $\left\langle\psi_{d}|D| \psi_{0}\right\rangle$ and $\left\langle\psi_{c}|D| \psi_{0}\right\rangle$, respectively. Note that the continuum state $\psi_{c}$ is energy-normalized and therefore has the dimension energy ${ }^{-1 / 2}$ whereas the discrete state $\psi_{d}$ is normalized to unity. The extra factor $\left(\frac{1}{2} \pi \Gamma\right)^{-1 / 2}$ that appears in the numerator of the expression (2) compensates for this, making $q$ dimensionless.

In a preceding paper [9] we have shown that contrary to what implicitly had been assumed in most papers on the subject, the majority of the $3 p \pi D$ state resonances in $\mathrm{H}_{2}$ are in fact "complex" resonances [10] resulting from many-level rovibronic interactions among Rydberg levels, and coupled to several continua of different type (ionization in addition to dissociation). This arises because for $v \geq 6$ the $3 p \pi D$ structures are embedded in the often dense manifold of higher $n p \sigma$ and $n p \pi$ levels and undergo rovibronic interactions with those. The Fano formula is designed to describe the interaction of a single isolated discrete eigenstate with a single flat continuum, and is obviously not appropriate for handling such situations. In many cases, however, the Fano formula may still be applied in a semi-quantitative fashion to the envelope of a "complex" resonance. Indeed, in some cases this has been done previously, as the complex nature of the resonances was simply not recognized due to insufficient spectral resolution or signal-to-noise ratio of the experiments.

By contrast, the lower vibration-rotation levels $v \leq 5$ of the $3 p \pi D$ state are rather well isolated levels and unperturbed in the sense of traditional molecular spectroscopy (except, obviously, to the extent that they are strongly predissociated). While the levels corresponding to $v=0,1$ and 2 lie below the $\mathrm{H}(1 s)+\mathrm{H}(n=2)$ threshold and are not predissociated, $v=3,4$ and 5 are predissociated and are good candidates for testing the Fano formula in detail, by comparing measured and predicted line shape parameters. Recently new high-resolution absorption measurements have been made on the DESIRS beamline of the SOLEIL synchrotron where many of the $D$ state predissociation resonances have been recorded with unprecedented spectral resolution [11]. We shall make use of some of these data in the present contribution, by specifically considering the $3 p \pi D^{1} \Pi_{u}, v=3, N=1, N=2$ and $N=3$ resonances with total parity $+(-1)^{N}$. Our predictions will be confronted in detail with the experimental data as well as with earlier calculations.

\section{MQDT: The $I+D$ approach}

The theoretical approach employed here has been developed over a number of years and its formal aspects have been discussed in a series of papers [12-17]. The approach is a combination of 
frame-transformation multichannel quantum defect theory (MQDT) and R-matrix techniques. We call it "I+D" since it allows one to calculate not only predissociation processes, " $\mathrm{D}$ ", but also their competition with molecular autoionization, "I", in situations where the total energy of the molecular system is higher than both the dissociation and ionization thresholds. Moreover, the theory accounts for non-adiabatic and/or electronic interactions that may take place among the dense manifolds Rydberg series of levels which are present at high excitation energy in any molecular system. Therefore the approach is not limited to the situation envisioned originally by Fano, but instead contains it as an extreme limiting case. We mention that the customary coupled differential equations ("CE") approach which is routinely applied to many problems of molecular dynamics by many research groups [18], is of course also nonperturbative and not limited to the assumptions made by Fano. However, as far as we are aware it has never been extended to the treatment of ionization in addition to dissociation processes.

An interesting point in this context is the fact that the $\mathrm{CE}$ approach is implemented in terms of a selected set of clamped-nuclei potential energy curves or surfaces, complemented by non-adiabatic interaction functions which couple the Born-Oppenheimer states and account for perturbations as well as dissociation processes. The variant of the "I+D" MQDT approach implemented here also starts out from clamped-nuclei potential energy curves, but it converts these to equivalent clamped-nuclei quantum defect functions. The quantum defects then constitute the basis for a frame transformation procedure which - while leaving aside the customary non-adiabatic coupling functions - provides directly rovibronic reaction matrices which account for non-adiabatic coupling as well as decay into ionization continua.

In the present calculations the manifold of ${ }^{1} \Sigma_{u}$ and ${ }^{1} \Pi_{u}$ excited states of $\mathrm{H}_{2}$ which can be reached by absorption of a photon are assumed to represent two singly excited $n p \sigma$ and $n p \pi$ Rydberg series converging to the $X^{2} \Sigma_{g}^{+}$ground state of $\mathrm{H}_{2}^{+}$. The electron $\sim$ core interaction for fixed nuclei corresponding to these two series is embodied in two energy- and $R$ - dependent quantum defect functions $\eta_{p}^{(\Lambda)}(E, R)(\Lambda=0$ and 1$) . \pi \eta_{p}^{(\Lambda)}(E, R)$ are the corresponding ion-electron scattering phase shifts which govern the molecular dynamics including ionization and dissociation processes. For the purposes of the present work these two functions have been extracted from state-of-the-art potential energy curves [19-21] for $n=2-4$ as has been discussed in detail in the Appendix of Ref. [9].

The present treatment of competing molecular ionization and dissociation processes is unified in the sense that the radial coordinate of the Rydberg electron, $r$, and the dissociative reaction coordinate, $R$, are treated on the same footing. Thus the concept of a "molecular core" is extended accordingly: it is defined by $r<r_{c}, R<R_{c}$, where $r_{c}$ corresponds to the range of the multipole and polarization long range fields of the molecular ion core, and $R_{c}$ corresponds, roughly, to the range of the molecular electronic ground and excited state potentials of the ion core. This latter parameter may be adjusted in accordance with the classical turning point of the vibrational level of the state of interest. Given the quantum defect functions, and for a selected value of $R_{c}$, an "I+D" type calculation then proceeds essentially as follows. For $R \leq R_{c}$, i.e. inside the range of the molecular potential, a rovibronic electronscattering calculation is carried out in the framework of frame-transformation MQDT, whereby at the boundary $R_{c}$ a uniform boundary condition (logarithmic derivative) is imposed on all vibrational wave functions that enter the calculation. The choice of this boundary condition does not affect those vibrational components that are vibrationally bound and do not reach out to $R_{c}$, typically for instance a $3 p \pi D, v, N$ level whose predissociation we are about to study. By contrast, the vibrational levels that are lying above a dissociation threshold or just below it are strongly affected by the choice of the boundary condition and/or the box size. Indeed, as the energy increases, they turn into particle-in-the-box states and, since a single fixed boundary condition has been imposed, their spectrum is discrete.

To the extent that these particle-in-the-box states are interacting with molecular levels that are confined to shorter $R$ - values, these latter also end up being somewhat dependent on the boundary condition. Figure 1 shows how this happens. Here, instead of varying the boundary condition imposed at $R_{c}$ we have varied $R_{c}$ itself - that is, we have varied the box size - while keeping the boundary 


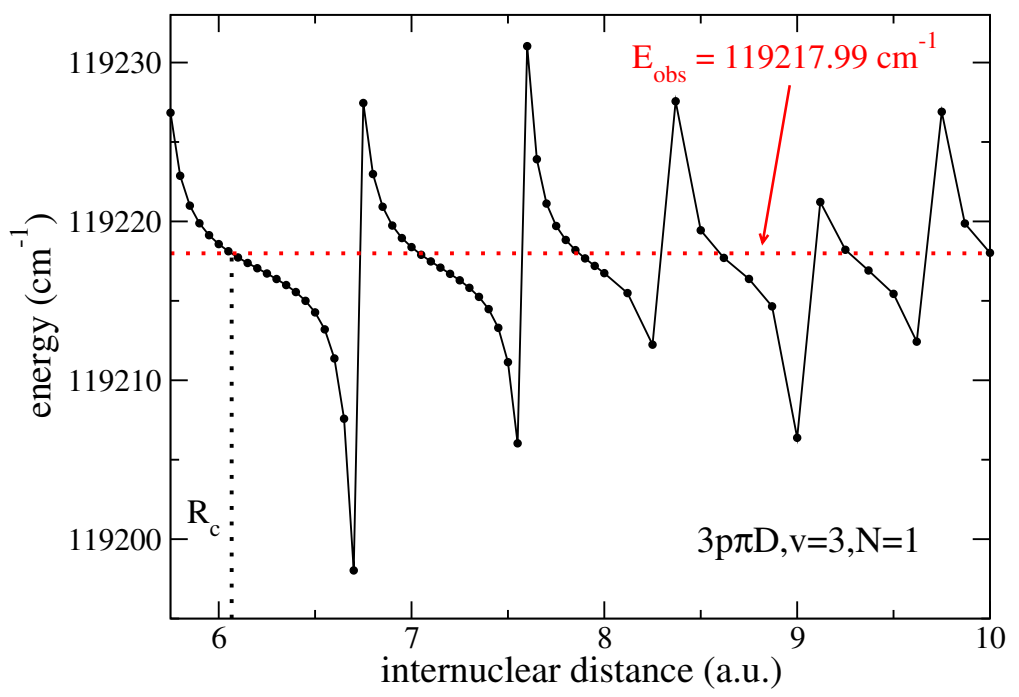

Figure 1. The energy of $3 p \pi^{1} \Pi_{u}^{+} D, v=3, N=1$ (negative total parity) level as a function of the R-matrix box size $R_{c}$. The black line with circles represents the standard MQDT calculations performed by applying a bound state type boundary conditions. The horizontal red dotted line represents the experimental value [11]. The vertical dotted line represents the $R_{c}$ value selected for the R-matrix calculation.

condition constant. (For instance, for the calculation shown in the figure all vibrational components were required to vanish at $R=R_{c}$.) The quantity plotted is the energy of the $3 p \pi D, v=3, N=1$ (negative total parity) level which is seen to exhibit a resonant pattern as functions of the value $R_{c}$, that oscillates around some mean value. For comparison the figure also shows the experimental position of the $3 p \pi D, v=3, N=1$ level from Ref. [11], and it may be seen that its position corresponds, inasmuch as may be judged by eye, to the average value of the resonance pattern.

Figure 2 displays another view of the same situation. Here we have plotted not just the energy of $3 p \pi, v=3, N=1$, but in addition also those of its nearest neighbor levels as function of the boundary condition (the negative of the logarithmic derivative imposed on the vibrational wavefunctions), for fixed $R_{c}$. The $3 p \pi D, v=3, N=1$ level appears as a nearly horizontal line because its vibrational wavefunction does not extend out to $R=R_{c}$. By contrast, the energies of the neighboring levels increase monotonically as the boundary condition $b=-\chi^{\prime} / \chi$ (with $\chi(R)$ any vibrational wavefunction) increases. Inspection of the MQDT wavefunctions of these extra levels indicates that they are discretized vibrational continuum quasi-levels of the $2 p \pi C$ and $3 p \sigma B^{\prime}$ states (represented in the figure by blue and red lines, respectively), whose dissociation asymptote lies at $118375.6 \mathrm{~cm}^{-1}[\mathrm{H}(1 s)+\mathrm{H}(n=2)$ threshold]. The saw-tooth pattern of Fig. 1 arises because each quasi-level interacts with $3 p \pi, v=$ $3, N=1$ as its energy is tuned through the bound-level position. This interaction leads to an avoided crossing, as shown in the inset of Fig. 2, whose nearest approach $2 V$ is a measure for the discrete $\sim$ continuum coupling and therefore for the predissociation width.

An estimate of the dissociation width $\Gamma$ may actually be obtained by making use of Fermi's "Golden Rule":

$$
\Gamma=2 \pi \frac{V^{2}}{\Delta E},
$$

where $\Delta E^{-1}$ is the density of the levels belonging to the quasi-continua. A rough estimate of the level density $\Delta E^{-1}$ may be obtained by just inspecting the quasi-level pattern shown in Fig. 2. More properly, it is evaluated by use of the phase-amplitude Milne approach as implemented in Ref. [22]. This approach 


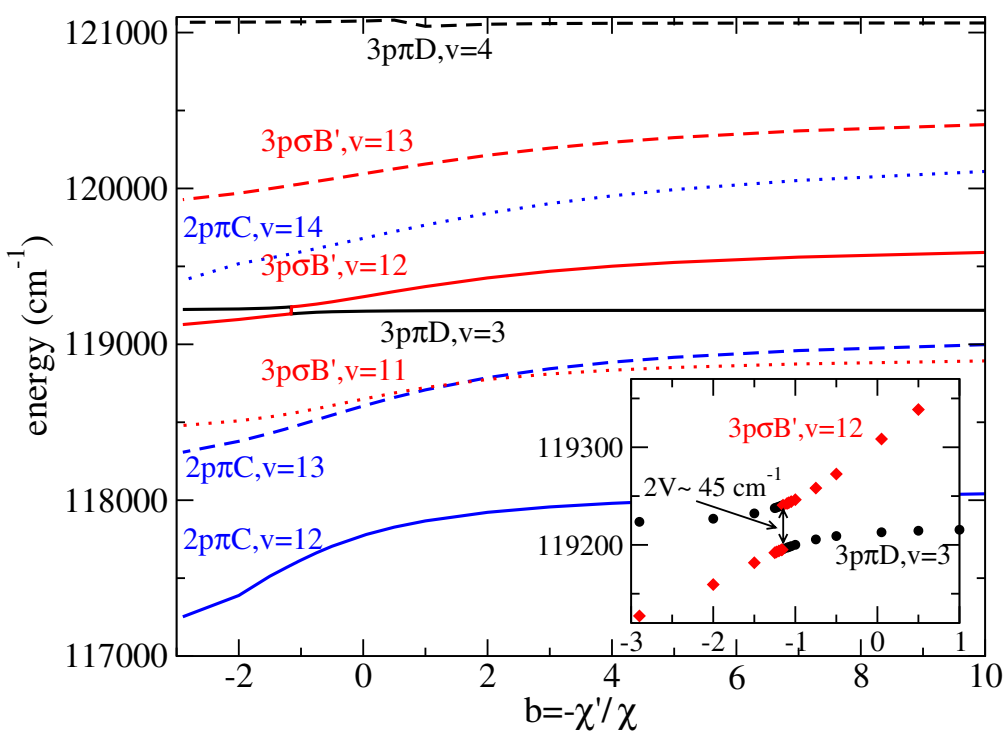

Figure 2. Energies of calculated singlet ungerade Rydberg levels with $N=1$ (negative total parity) in $\mathrm{H}_{2}$ plotted as functions of the boundary condition $b$ applied to the vibrational basis functions. The black curves represent the $3 p \pi D, v=3$ and $v=4$ levels, while the red curves represent the $3 p \sigma B^{\prime}, v=11-13$ levels and the blue ones represent the $2 p \pi C, v=12-14$ levels, respectively. The inset displays the avoided crossing that occurs as a result of the vibronic interaction between $3 p \pi D, v=3$ and the quasi-level $3 p \sigma B^{\prime}, v=12$ which is part of the discretized dissociation continuum.

permits the evaluation of the phase $\phi\left(R_{c}\right)$ of the vibrational wavefunction, $\chi(R) \approx \alpha(R) \sin \phi(R)$, associated with the potential of the $2 p \pi C$ dissociating state, accumulated between $R=0$ and $R=R_{c}$. This may be done for arbitrary energy $E$, that is, in the present case, the energy at which the avoided crossing occurs. By calculating the accumulated phase for two energies separated by an infinitesimal increment the rate of change of the accumulated phase may be obtained. The level density $\Delta E^{-1}$ is the inverse of this quantity. Equation (3) has been used in Refs. [23] and [24] for the purpose of estimating predissociation widths, but in order to map out the details of the resonances and account for partial cross sections the full approach of Refs. [12-17] must be implemented. This involves the proper continuation of the wavefunctions associated with the quasi-levels seen in Fig. 2, into the asymptotic range beyond $R=R_{c}$, using the full panoply of scattering theory. We do not discuss these aspects in detail here, but refer the reader to the references given.

\section{Results}

The I+D/MQDT calculations reported here have been carried out in the same manner as those published in Ref. [9]. The input data (quantum defects, channel dipole transition moments, $\mathrm{H}_{2}^{+} \mathrm{PE}$ curve, ion core adiabatic corrections, mass correction to the quantum defects), the vibrational basis sets employed, as well as the numerical implementation are exactly as described there.

\subsection{Fitting I+D/MQDT profiles with the Fano formula}

From the work of Breit and Wigner [8] we know that near an isolated resonance the eigenphase $\pi \tau$ (or eigenphase sum, if several continua are open) will increase by an amount of $\pi$ as function of the 

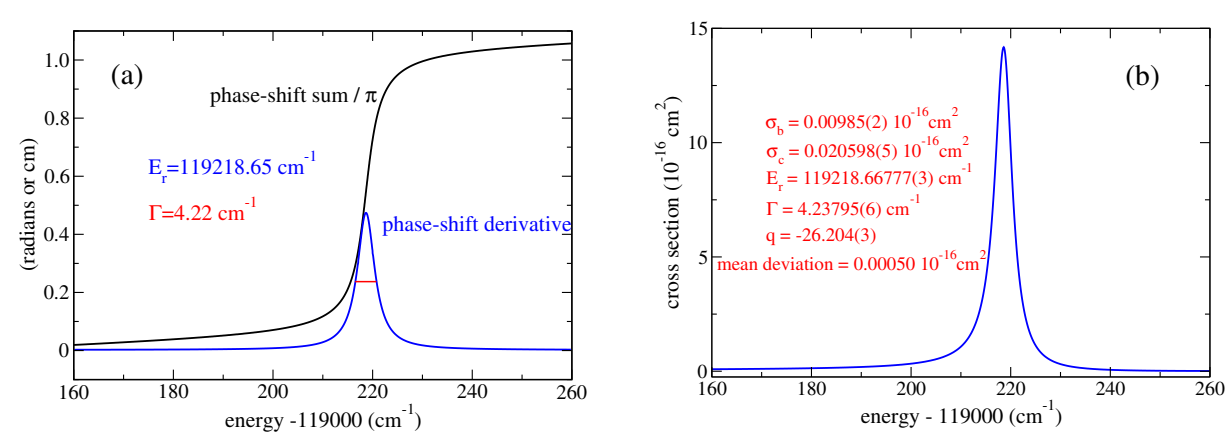

Figure 3. I+D/MQDT calculation of the dissociation phase-shift sum and its energy derivative as a function of the energy near the predissociated level $3 p \pi D, v=3, N=1$ (negative total parity). a) The black curve is the phaseshift sum divided by $\pi$, while the blue curve is its energy-derivative. The resonance position and width derived from the phase-shift derivative are indicated. b) The blue curve is the absorption profile from the $v^{\prime \prime}=0$ ground state level calculated with the I+D/MQDT approach $(R(0)$ transition). This profile has been fitted to the Fano profile Eq. (1). The resulting fit parameters and the mean deviation are indicated in the figure.

energy, with the analytical dependence given by $\tan \pi \tau(E)=-(\Gamma / 2) /\left(E-E_{r}\right)$. The energy derivative $\partial(\pi \tau) / \partial E$ thus corresponds to a Lorentzian centered at $E_{r}$ with a peak value $2 / \Gamma$ and a width $\Gamma$ (FWHM). The knowledge of the phase therefore allows us to extract the resonance position $E_{r}$ and width $\Gamma$ directly from the excited state scattering wavefunction of the resonance without passing through the photoabsorption intensity profile. The latter may then be used subsequently to extract the remaining three parameters entering Eqs. (1) and (2), namely the non-resonant and resonant background cross sections $\sigma_{b}$ and $\sigma_{c}$, as well as the shape parameter $q$.

Figure 3 demonstrates how this has been done for the $3 p \pi D, v=3, N=1$ (negative total parity) resonance. Figure $3 \mathrm{a}$ shows how the resonance position and width are extracted from the energydependent continuum phase. The values thus obtained are indicated in the figure. Figure $3 \mathrm{~b}$ shows what is obtained when all parameters of the Fano profile formula are extracted by a least-squares procedure directly from the theoretical intensity line profile calculated by I+D/MQDT. The parameters obtained are again included in the figure. It may be seen that the theoretical profile perfectly fits the Fano line shape, to the extent that the two cannot be distinguished on the scale of Fig. 3b. The formal uncertainties are far smaller than the accuracy we can expect from our I+D/MQDT calculation as a whole. This means that for all practical purposes the $R(0) v=3 D$ state absorption profile may indeed be considered to be a Fano profile. Note also that the energy range over which we have fitted the I+D/MQDT profile is quite large as it spans a range of 50 units in terms of the reduced energy $\epsilon$ [Eq. (2)]. Interestingly, we find that the deviations in the wings of the resonance are larger than those in the center despite the fact that the intensity there is much smaller than in the center. On the other hand, the comparison of the resonance positions $E_{r}$ and widths $\Gamma$ derived in two different ways in Figs. 3a and 3b shows differences of about $0.02 \mathrm{~cm}^{-1}$ for both parameters which is not quite negligible in terms of the numerical accuracy achieved here (the accuracy of the quantum defects employed is another, independent, matter which does not come in here). Figure 4 extends this analysis to the $R(1)$ and $R(2)$ transitions. The quality of the fits of the I+D/MQDT profiles by Eq. (1) are similar to those obtained for the $R(0)$ transition but the widths do again not correspond exactly to those derived from the energy dependence of the dissociation phase.

As a conclusion of this subsection - inasmuch as a conclusion may be drawn from just a few examples - it appears that, while the Fano profile formula provides a surprisingly accurate representation of isolated resonance shapes in a limited energy range, the parameters that go into it may have a slightly empirical character rather than a precise meaning in terms of quantum mechanics. In other words, the 


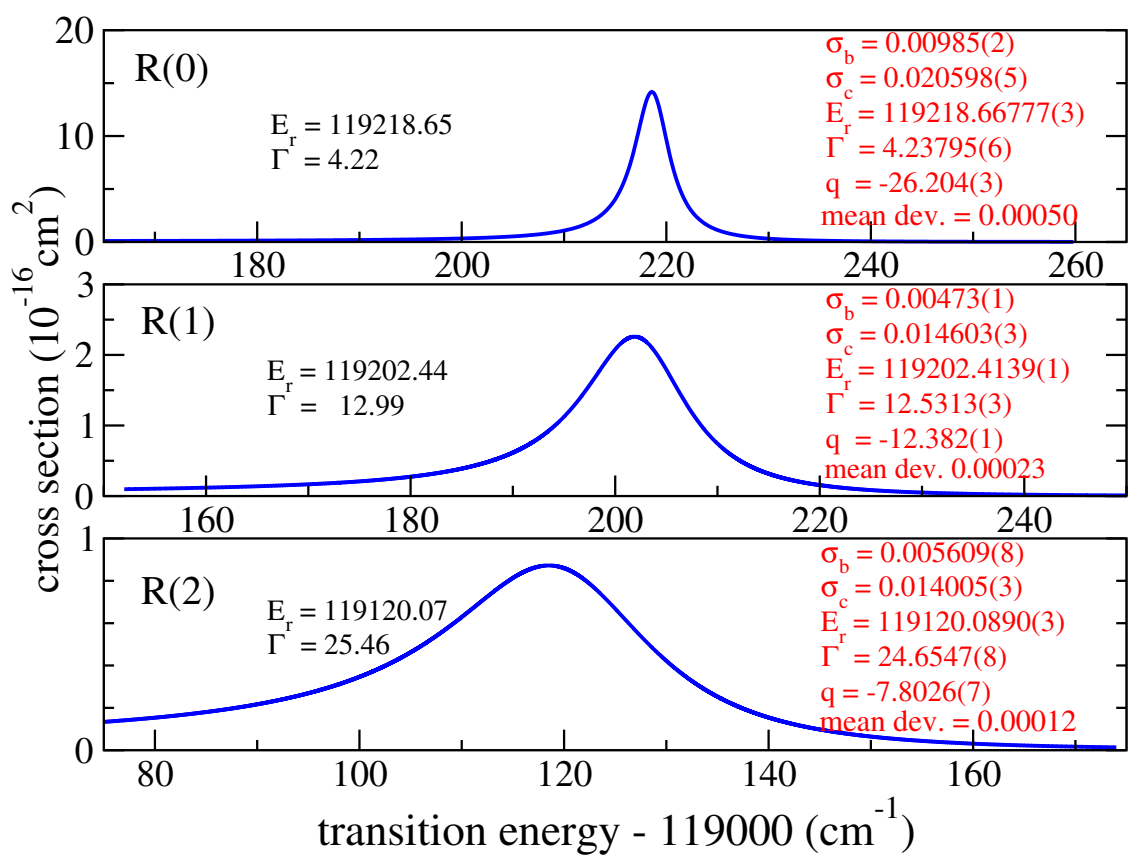

Figure 4. Blue curves: $R(0), R(1)$ and $R(2)$ absorption profiles of the $3 p \pi^{1} \Pi_{u}^{+}, v=3 \leftarrow X^{1} \Sigma_{g}^{+}, v^{\prime \prime}=0$ transition in $\mathrm{H}_{2}$ calculated with the I+D/MQDT approach. Each profile has been fitted to the Fano profile Eq. (1). The resulting fit parameters and the mean deviation are indicated in red. The units are the same as in Fig. 3b. The resonance positions $E_{r}$ and widths $\Gamma$ derived from the eigenphase sum of the MQDT calculation are indicated in black.

values of the parameters may not necessarily convey exactly the meaning they were given in Fano's original derivation [Eq. (2)]. The comments on this point made by Comes and Sälzer [5] and Fano and Cooper [25] go more or less in the same direction.

\subsection{Prediction of observed profiles with theoretical Fano parameters}

In view of what has just been said it appears that the most conclusive test of theory is not so much to compare predicted resonance positions, widths and shape parameters with corresponding values derived from experiment, but to proceed by the inverse route, by directly comparing predicted spectral profiles with their observed counterparts. We do this in the following for the $R(0)$ and $R(1)$ transitions of the $3 p \pi D^{1} \Pi_{u}^{+}, v=3 \leftarrow X^{1} \Sigma_{g}^{+}, v^{\prime \prime}=0$ transition which lie very close to each other and actually overlap in the spectrum. The most complete and accurate previous computations of these spectral lines where transition probabilities have been evaluated in addition to the predissociation process itself, are those of Beswick and Glass-Maujean [26] and Mrugała [27]. Both studies were based on the coupled equations (CE) approach, and in both papers the resulting profiles have been analyzed in terms of Fano's profile formula.

We compare the theoretical cross section Fano profiles with the Fourier transform absorption spectrum from Ref. [11]. To this end we take account of the temperature of $100 \mathrm{~K}$ used in that experiment, and the fact that no ortho para conversion occurs as the $\mathrm{H}_{2}$ gas is cooled down rapidly from room temperature (see [9] for details). The relevant parameters for implementing Eqs. (1) and (2) may be taken directly from Table 1 of Ref. [27]. In the case of Ref. [26] only $\Gamma$ and $q$ are given in numerical form, whereas $\sigma_{c}$ and $E_{r}$ had to be extracted from the positions and intensities of the 


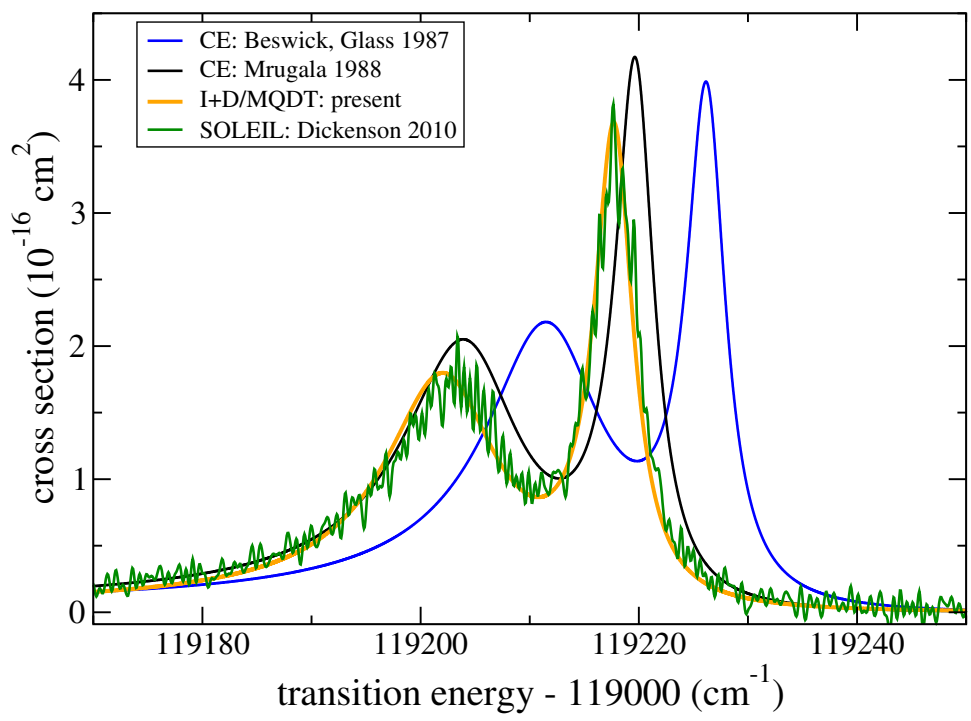

Figure 5. $R(0)$ and $R(1)$ resonance profiles of the $3 p \pi^{1} \Pi_{u}^{+}, v=3 \leftarrow X^{1} \Sigma_{g}^{+}, v^{\prime \prime}=0$ transition in $\mathrm{H}_{2}$, recorded on the DESIRS beamline at the SOLEIL synchrotron by Dickenson et al. [11], are compared with ab initio Fano profiles obtained with the coupled equations (CE) approach by Beswick and Glass-Maujean [26] and Mrugała [27] and by the present I+D/MQDT approach. The theoretical spectra have been synthetized by assuming a $\mathrm{H}_{2}$ gas temperature of $100 \mathrm{~K}$ with an ortho-para distribution corresponding to room temperature.

peak maxima displayed in their Fig. 8. According to Eq. (1), the position $\epsilon_{\max }$ and intensity $\sigma_{\max }$ are given by

$$
\begin{aligned}
\epsilon_{\max } & =\frac{1}{q} \\
\sigma_{\max } & =\sigma_{b}+\sigma_{c}\left(1+q^{2}\right),
\end{aligned}
$$

allowing us to obtain the desired parameters easily and quite accurately via Eq. (2) by setting $\sigma_{b}$ to zero. Based on these considerations, theoretical spectra have been synthesized by superposing the $R(0)$ and $R(1)$ Fano profiles.

The comparison between the different theoretical predictions and the SOLEIL experiment is illustrated by Fig. 5. The figure shows that the overall visual agreement between experiment and theory appears the better the more recent is the calculation. Note however, that the improvement concerns mainly the line positions, whereas the profiles as such were rendered essentially perfectly already by the computations made more than 25 years ago. The improved agreement of the line positions is no doubt due mainly to the fact that improved potential energy curves have become available more recently, such as used in the present calculations, which are based on the most recent $a b$ initio data from Wolniewicz and coworkers [19-21]. In an earlier paper that dealt with the purely spectroscopic aspects of the $\mathrm{H}_{2}$ absorption spectrum [28] - line positions of sharp lines essentially - we found that for low- $n$ states $n=2$ and 3 - MQDT yields results that are comparable, but hardly better, than those obtained with the CE approach. This comparison was based on identical PEC input data from Refs. [19-21]. The advantage of MQDT is that it yields a global description of channel interactions over a considerable range of energies, extending into regions where complex perturbations among Rydberg series arise, autoionization enters into competition with predissociation, and the $\mathrm{CE}$ approach no longer offers a viable alternative. 
The peak intensities of the three calculations included in Fig. 5 agree to within about $10 \%$. As to the best of our knowledge no absolute intensity measurements exist for the transitions to the $3 p \pi D, v=3$ state, we have adjusted the experimental data by application of an overall scaling factor for the purposes of the comparison. This adjustment is somewhat biased as we have used our own calculation for the calibration. Our previous paper [9] has shown that for $v=6$ and 8 where an absolute calibration of the SOLEIL spectra has been possible, our theoretical peak intensities match the experimental values to within a few percent.

We conclude this subsection by saying that the I+D/MQDT method implemented purely with firstprinciples input parameters is able to reproduce the $v=3$ predissociation peak positions to within $<1 \mathrm{~cm}^{-1}$ and the lineshapes with very high accuracy. The parametrization in terms of Fano's line shape formula leads to no loss of accuracy.

\section{Conclusion}

We have examined first principles coupled equations (CE) [26, 27] and multichannel quantum defect theory (I + D/MQDT, present work) predictions for the $3 p \pi D^{1} \Pi_{u}^{+}, v=3 \leftarrow X^{1} \Sigma_{g}^{+}, v^{\prime \prime}=0 R(0), R(1)$ and $R(2)$ predissociation resonances. We have found that they can be parametrized perfectly in terms of the Fano line shape formula Eq. (1). The test was carried out over a range of $\approx 100 \mathrm{~cm}^{-1}$ in each case, corresponding to a multiple of the resonance width. The experimental line shapes of these transitions, recorded recently under high resolution at the SOLEIL synchrotron [11], are very well reproduced by all three computations, but the resonance positions are rendered best by the present calculations.

We are indebted to the French Agence Nationale pour la Recherche and the Triangle de la Physique for generous financial support in the framework of the 09-BLAN-02090 SUMOSTAI and SpeCoRyd contracts. J.Zs.M. is grateful for financial support also from the Région de Haute Normandie (CPER THETE project) and the LabeX EMC $^{3}$ (PicoLIBS project). Ch.J. is indebted to the Miescher Foundation (Basel, Switzerland) for partial support. Finally, the authors acknowledge travel support from the International Atomic Energy Agency (IAEA) via two of their Co-ordinated Research Projects (CRP).

\section{References}

[1] H. Beutler, A. Deubner and H. Jünger, Z. Phys. 98, 181 (1935)

[2] H. Beutler, Z. Phys. 93, 177 (1935)

[3] U. Fano, Nuovo Cimento, 12, 156 (1935)

[4] U. Fano, Phys. Rev. 124, 1866 (1961)

[5] F. J. Comes and H. G. Sälzer, Phys. Rev. 152, 29 (1966)

[6] M. Wickenhauser, J. Burgdörfer, F. Krausz, and M. Drescher, Phys. Rev. Lett. 94, 023002 (2005)

[7] C. Ott, A. Kaldun, P. Raith, K. Meyer, M. Laux, J. Evers, C. H. Keitel, C. H. Greene, and T. Pfeifer, Science 340, 716 (2013)

[8] G. Breit and E. Wigner, Phys. Rev. 49, 519 (1936)

[9] J. Zs. Mezei, I. F. Schneider, M. Glass-Maujean, and Ch. Jungen, J. Chem. Phys. 141, 064305 (2014)

[10] Ch. Jungen and M. Raoult, Farad. Disc. Chem. Soc. 71, 253 (1981)

[11] G. D. Dickenson, T. I. Ivanov, M. Roudjane, N. de Oliveira, D. Joyeux, L. Nahon, W.-Ü. L. Tchang-Brillet, M. Glass-Maujean, I. Haar, A. Ehresmann, and W. Ubachs, J. Chem. Phys. 133, 144317 (2010)

[12] Ch. Jungen, Phys. Rev. Lett. 53, 2394 (1984)

[13] C. H. Greene and Ch. Jungen, Adv. At. Mol. Phys. 21, 51 (1985)

[14] H. Gao, Ch. Jungen and C. H. Greene, Phys. Rev. A 47, 4877 (1993) 
[15] Ch. Jungen and S. C. Ross, Phys. Rev. A 55, R2503 (1997)

[16] A. Matzkin, Ch. Jungen and S. C. Ross, Phys. Rev. A 62, 062511 (2000)

[17] Ch. Jungen, Elements of Quantum Defect Theory, edited by M. Quack and F. Merkt, Handbook of High Resolution Spectroscopy, (Wiley, Chichester and New York, 2011)

[18] see e.g. D. Secrest, in Atom-Molecule Collision Theory, A Guide for the Experimentalists, edited by R. B. Bernstein (Plenum, New York, 1979), p. 265

[19] L. Wolniewicz and G. Staszewska, J. Mol. Spectrosc. 212, 208 (2002)

[20] L. Wolniewicz and G. Staszewska, J. Mol. Spectrosc. 220, 45 (2003)

[21] A compilation of the numerical data published in Refs. [19, 20] can be found in http://www.fizyka.umk.pl/publications/ifiz/luwo/

[22] Ch. Jungen and F. Texier, J. Phys. B: At. Mol. Opt. Phys. 33, 2495 (2000)

[23] M. Glass-Maujean, Ch. Jungen, G. Reichardt, A. Balzer, H. Schmoranzer, A. Ehresmann, I. Haar and P. Reiss, Phys. Rev. A 82, 062511 (2010)

[24] M. Glass-Maujean, H. Schmoranzer, Ch. Jungen, I. Haar, A. Knie, P. Reiss and A. Ehresmann, Phys. Rev. A 86, 052507 (2012)

[25] U. Fano and J. W. Cooper, Rev. Mod. Phys. 40, 441 (1968)

[26] J. A. Beswick and M. Glass-Maujean, Phys. Rev. A 35, 3339 (1987)

[27] F. Mrugała, Mol. Phys. 65, 377 (1988)

[28] M. Glass-Maujean, Ch. Jungen, M. Roudjane, and W.-Ü L. Tchang-Brillet, J. Chem. Phys. 134, 204305 (2011) 